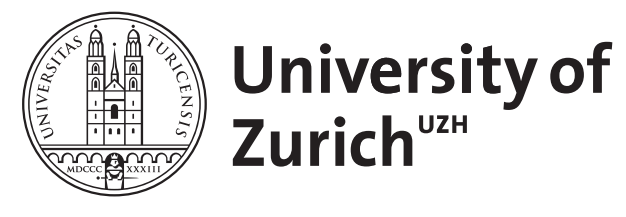

\title{
Regular selections for multiple-valued functions
}

De Lellis, C ; Grisanti, C ; Tilli, P

\begin{abstract}
Given a multiple-valued function f, we deal with the problem of selecting its single valued branches. This problem can be stated in a rather abstract setting considering a metric space E and a finite group $\mathrm{G}$ of isometries of $\mathrm{E}$. Given a function $\mathrm{f}$ which takes values in the equivalence classes of $\mathrm{E} / \mathrm{G}$, the problem consists in finding a map $g$ with the same domain as $\mathrm{f}$ and taking values in E, such that at every point $t$ the equivalence class of $g(t)$ coincides with $f(t)$. If the domain of $f$ is an interval, we show the existence of a function $g$ with these properties which, moreover, has the same modulus of continuity of $\mathrm{f}$. In the particular case where $\mathrm{E}$ is the product of $\mathrm{Q}$ copies of $\mathrm{n}$ and $\mathrm{G}$ is the group of permutations of $\mathrm{Q}$ elements, it is possible to introduce a notion of differentiability for multiple valued functions. In this case, we prove that the function $\mathrm{g}$ can be constructed in such a way to preserve $\mathrm{C} \mathrm{k}$, regularity. Some related problems are also discussed.
\end{abstract}

DOI: https://doi.org/10.1007/s10231-003-0081-5

Posted at the Zurich Open Repository and Archive, University of Zurich

ZORA URL: https://doi.org/10.5167/uzh-21813

Journal Article

Originally published at:

De Lellis, C; Grisanti, C; Tilli, P (2004). Regular selections for multiple-valued functions. Annali di Matematica Pura ed Applicata, 183(1):79-95.

DOI: https://doi.org/10.1007/s10231-003-0081-5 


\title{
REGULAR SELECTIONS FOR MULTIPLE VALUED FUNCTIONS
}

\author{
CAMILLO DE LELLIS ${ }^{1}$,CARLO ROMANO GRISANTI ${ }^{2}$, PAOLO TILLI $^{3}$
}

\begin{abstract}
Given a multiple valued function $f$, we deal with the problem selecting its single valued branches. This problem can be stated in a rather abstract setting considering a metric space $E$ and a finite group $G$ of isometries of $E$. Given a function $f$ which takes values in the equivalence classes of $E / G$, the problem consists in finding a map $g$ with the same domain as $f$ and taking values in $E$, such that at every point $t$ the equivalence class of $g(t)$ coincides with $f(t)$.

If the domain of $f$ is an interval, we show the existence of a function $g$ with these properties which, moreover, has the same modulus of continuity of $f$. In the particular case where $E$ is the product of $Q$ copies of $\mathbb{R}^{n}$ and $G$ is the group of permutations of $Q$ elements, it is possible to introduce a notion of differentiability for multiple valued functions. In this case, we prove that the function $g$ can be constructed in such a way to preserve $C^{k, \alpha}$ regularity.

Some related problems are also discussed.
\end{abstract}

\section{INTRODUCTION}

The theory of multiple valued functions in the sense of Almgren (see [2]) has several applications in the framework of geometric measure theory. Indeed, multiple valued functions represent a very effective tool to approximate more abstract objects arising in geometric measure theory. For example, Almgren (see [2]) shows that some rectifiable currents are approximated by the graph of Lipschitz multiple valued functions. A special class of varifolds with second fundamental form in $L^{p}$, introduced by Hutchinson (see [12]), can be locally represented by the graph of a multiple valued function. There are also other objects similar to these functions, like the union of Sobolev's functions graphs

\footnotetext{
${ }^{1}$ Scuola Normale Superiore, Piazza dei Cavalieri 7, 56100 Pisa (Italy) e-mail: delellis@cibs.sns.it

${ }^{2}$ Dipartimento di Matematica Applicata "U. Dini", Univesità di Pisa via Bonanno Pisano 25B, 56126 Pisa (Italy)

e-mail: grisanti@dma.unipi.it

${ }^{3}$ Scuola Normale Superiore, Piazza dei Cavalieri 7, 56100 Pisa (Italy) e-mail:paolo@tilli.sns.it
} 
introduced by Ambrosio, Gobbino \& Pallara (see [6]), or the weak limits of union of graphs of $C^{\alpha}$ functions proposed by De Giorgi (see $[8,9])$. The relations between these objects and Hutchinson's varifolds are investigated in [6].

A general abstract setting to deal with multiple valued functions is the following (see Section 2 for details). Given a metric space $(E, d)$ and a finite group $G$ of isometries of $E$, the quotient space $E / G$ can be endowed with the quotient metric $\tilde{d}$ in a natural way. Moreover, for every $x \in E$ we denote by $[x] \in E / G$ the orbit of $x$ under $G$, i.e. the set $\{\tau x\} \subset E$ as $\tau$ ranges through $G$.

Given a map $f: A \mapsto E / G$, where $A$ is some set, the selection problem for $f$ is the following: to find $\tilde{f}: A \mapsto E$ such that $[\tilde{f}(t)]=f(t)$ holds for every $t \in A$. It is clear that this problem can always be solved by virtue of the Zorn Lemma: in the following, however, we shall be concerned with the existence of a selection which preserves some properties of $f$ such as continuity, but also differentiability and higher regularity (provided, of course, that the structure of $E$ allows some notion of smoothness).

In the case where the set $A$ is an interval, we can prove that every continuous path admits a selection which inherits the modulus of continuity:

Theorem 1.1. Let $(E, d)$ be a metric space and let $G$ be a finite group of isometries of $E$. Suppose $f:[0,1] \rightarrow E / G$ is a continuous curve, and let $\omega_{f}$ denote the modulus of continuity of $f$, i.e.

$$
\omega_{f}(\delta):=\sup \{\tilde{d}(f(t), f(s)): t, s \in[0,1] \text { and }|t-s| \leq \delta\} .
$$

Then there exists a selection $g:[0,1] \rightarrow E$ of $f(i . e .[g(t)]=f(t)$ for every $t \in[0,1]$ ) such that

$$
\omega_{g} \leq C_{G} \omega_{f},
$$

where $\omega_{g}$ denotes the modulus of continuity of $g$ and $C_{G}$ is a constant which depends only on the order of $G$.

The connection with multiple valued functions becomes apparent if $E=X^{Q}$ is the product of $Q$ copies of a metric space $X$ and $G$ is the symmetric group of order $Q$, acting on $X^{Q}$ in the natural way, i.e. if $e=\left(x_{1}, \ldots, x_{Q}\right) \in X^{Q}$ and $\tau \in G$ is a permutation, then

$$
\tau e=\left(x_{\tau_{1}}, \ldots, x_{\tau_{Q}}\right) .
$$

In this case, the quotient space $E / G$ is just the set of all unordered $Q$ tuples of elements of $X$, repetitions being allowed. A function $f$ with values in $E / G$ is then multiple valued in the usual sense, and solving 
the selection problem for $f$ means isolating $Q$ single valued branches of $f$.

Particularly relevant in the applications is the case where $X=\mathbb{R}^{n}$, hence $E=\left(\mathbb{R}^{n}\right)^{Q}$ and $E / G$ is the space of all unordered $Q$-tuples of vectors of $\mathbb{R}^{n}$, repetitions being allowed. Keeping the notation of Hutchinson [12], we denote this space of $Q$-tuples by $\mathbf{Q}_{Q}\left(\mathbb{R}^{n}\right)$.

In this case, due to the linear structure of $\mathbb{R}^{n}$, there is a natural notion of multiple valued function of class $C^{k, \alpha}$, which reduces to the usual one when $Q=1$ (see Sections 3 and 4 for details). It turns out that the selection problem for a $C^{k, \alpha}$ multiple valued function can be solved preserving $C^{k, \alpha}$ regularity. Indeed, in Section 4 we will prove the following result.

Theorem 1.2. Let $f:[a, b] \longrightarrow \mathbf{Q}_{Q}\left(\mathbb{R}^{n}\right)$ be a $C^{k, \alpha} Q$-valued function. Then there exist functions $g_{i}:[a, b] \longrightarrow \mathbb{R}^{n}$ such that $g_{i} \in C^{k, \alpha}([a, b])$ for $i=1, \ldots, Q$, and the $Q$-tuple $f(x)$ coincides with $\left\{g_{i}(x)\right\}_{i=1}^{Q}$ for every $x \in[a, b]$.

The proof of this theorem consists of two steps. First we prove that a multivalued continuous function can be split into single branches which inherit the modulus of continuity. Then we are able to recover differentiability thanks to the last theorem of Section 3. The first step, of course, relies on Theorem 1.1 above.

Some classical statement and definitions about multivalued functions in $\mathbb{R}^{n}$ are recalled in Section 3, whereas in the last section of the paper we will give a partial result when the domain of $f$ is $\mathbb{R}^{m}$ and it takes values in the $Q$-tuples of real numbers. In this case we prove that if $f$ is continuous, then it is a finite union of continuous branches, but we are not able to extend this result to regularity higher than mere continuity.

\section{LiFTING OF PATHS}

This section is entirely devoted to the proof of Theorem 1.1.

Let $(E, d)$ be a metric space, and let $G$ be a finite group of isometries of $E$. Given $x \in E$, we define the orbit of $x$ as

$$
[x]:=\bigcup_{\tau \in G}\{\tau x\} .
$$

It is clear that the relation

$$
x \sim y \Leftrightarrow[x]=[y]
$$

is an equivalence relation, and $x \sim y$ holds true if and only if $x=\tau y$ for some $\tau \in G$. Moreover, the quotient space $E / \sim$ of the equivalence 
classes (denoted also by $E / G$ ) can be given a natural metric in the following way:

$$
\tilde{d}([x],[y]):=\min _{\sigma \in G} d(x, \sigma y) .
$$

Given a set $A$ and a function $f: A \rightarrow E / G$, a function $g: A \rightarrow E$ is said to be a selection (or also a lifting) of $f$ if

$$
[g(t)]=f(t) \quad \forall t \in A .
$$

The proof of Theorem 1.1 can be divided into three steps.

(a) First we consider the case where $E$ is a vector space: the general case will later be recovered by an embedding argument;

(b) we construct a sequence of equicontinuous and piecewise affine functions $\left(g_{n}\right)$, which sort of interpolate $f$ on a mesh of points;

(c) we use a variant of the classical Ascoli-Arzelà Theorem to show that the sequence of functions has a cluster point and we show that this limiting function is a selection for $f$.

On estimating the modulus of continuity of $g_{n}$ in step (b), we rely the following combinatorial lemma about finite groups which, to the best of our knowledge, cannot be found in the literature.

Lemma 2.1. Let $G$ be a finite group of order $o(G)$ and let $m>0$ be a natural number. Suppose that $\left\{\sigma_{i, j}\right\}$, with $0 \leq i<j \leq m$, are elements of $G$, with the only assumption that

$$
\sigma_{i, i+1}=1_{G}, \quad i=0, \ldots, m-1 .
$$

Then there exist a natural number $k$ with $k \leq 2 \mathrm{o}(G)-1$, and natural numbers $\left\{i_{j}\right\}, j=0, \ldots, k$ such that

$$
0=i_{0}<i_{1}<\cdots<i_{k-1}<i_{k}=m
$$

and

$$
\sigma_{i_{0}, i_{1}} \sigma_{i_{1}, i_{2}} \cdots \sigma_{i_{k-1}, i_{k}}=1_{G}
$$

Before proceeding to the proof, we introduce some terminology. Under the assumption of Lemma 2.1, we say that $\tau \in G$ is $h$-reachable in (at most) s steps if there exist $\left\{i_{j}\right\}, j=0, \ldots, r$ such that $r \leq s$,

$$
0=i_{0}<i_{1}<\cdots<i_{r}=h
$$

and

$$
\sigma_{i_{0}, i_{1}} \sigma_{i_{1}, i_{2}} \cdots \sigma_{i_{r-1}, i_{r}}=\tau \text {. }
$$

If $E \subseteq G$, we say that $E$ is $h$-reachable in $s$ steps if each of its elements is. We note that if $E$ is $i$-reachable in $s$ steps, then $E \sigma_{i, j}$ (where $E \sigma$ denotes the set $\{\tau \sigma\}$ as $\tau$ runs through $E$ ) is $j$-reachable in $s+1$ steps, whenever $i<j \leq m$. 
With this terminology, Lemma 2.1 states that $1_{G}$ is $m$-reachable in at most $2 \circ(G)-1$ steps (note that, due to (4), $1_{G}$ is trivially $m$-reachable in $m$ steps: therefore, the nontrivial part of the lemma is an upper bound to $k$ which is independent of $m$ ).

Proof. If $G=\left\{1_{G}\right\}$ or $m=1$, then the lemma is trivial, so we suppose that $|G|>1$ and $m>1$. Let $\mathcal{P}$ be the family of all pairs $(i, E)$ such that $1<i \leq m, 1_{G} \in E \subseteq G,|E| \geq 2$ and

$$
E \text { is } i \text {-reachable in at most } 2(|E|-1) \text { steps. }
$$

Let us partially order $\mathcal{P}$ by monotonicity, i.e.

$$
(i, E) \leq(j, F) \Leftrightarrow i \leq j \text { and } E \subseteq F .
$$

Step 1. If $\mathcal{P}=\emptyset$, then the lemma holds true.

To see this, define

$$
I:=\left\{j: 1<j \leq m \text { and } \sigma_{0, j} \neq 1_{G}\right\} .
$$

If $I=\emptyset$, then $\sigma_{0, m}=1_{G}$ and the claim follows. Otherwise, we set $i:=\min I$ and $E=\left\{1_{G}, \sigma_{0, i}\right\}$. But then $(E, i) \in \mathcal{P}$, since $\sigma_{0, i}$ is trivially $i$-reachable in one step, and $\sigma_{0, i-1} \sigma_{i-1, i}=\sigma_{0, i-1}=1_{G}$ (we have used the definition of $i$ and (4)), hence $1_{G}$ is $i$-reachable in two steps.

Step 2. If $\mathcal{P} \neq \emptyset$, let $(i, E) \in \mathcal{P}$ be maximal (with respect to the above partial order). If $i=m$, then $1_{G} \in E$ is $m$ reachable in $2|E|-2<$ $2 \mathrm{o}(G)-1$ steps and the claim follows. If $i<m$ and $E=G$, then $\sigma_{i, m}^{-1} \in E$ is $i$-reachable in $2 \mathrm{o}(G)-2$ steps, hence $1_{G}$ is $m$ reachable in $2 \mathrm{o}(G)-1$ steps and the claim follows.

It remains to consider the case where $i<m$ and $E \subset G$ with strict inclusion. In this case, let

$$
I:=\left\{j: i<j \leq m \text { and } E \sigma_{i, j} \neq E\right\} .
$$

If $I=\emptyset$, then $E \sigma_{i, m}=E$; in particular, $e \sigma_{i, m}=1_{G}$ for some $e \in E$. Since $e$ is $i$-reachable in $2|E|-2$ steps, $1_{G}$ is $m$-reachable in $2|E|-1$ steps and the claim follows.

If $I \neq \emptyset$, we define $j:=\min I$ and $F:=E \cup E \sigma_{i, j}$. If we show that $(j, F) \in \mathcal{P}$, then we get a contradiction (since this would violate the maximality of $(i, E))$ and the lemma is completely proved. It suffices to show that the whole $F$ is $j$-reachable in $2|F|-2$ steps. Not that (4) implies that $j>i+1$, hence the choice of $j$ implies $E \sigma_{i, j-1}=E$, and therefore $E \sigma_{i, j-1} \sigma_{j-1, j}=E$. Using (7), we obtain that $E$ is $j$-reachable in $2|E|$ steps. Similarly, $E \sigma_{i, j}$ is $j$-reachable in $2|E|$ steps, and the proof is completed observing that $2|E| \leq 2|F|-2$ (note the $|F|>|E|$, since $\left.E \sigma_{i, j} \neq E\right)$. 
Now we are in a position to prove Theorem 1.1 in full details.

Proof of Theorem 1.1. We first suppose that $E$ is a normed vector space (and hence that $d(x, y)=\|x-y\|$ ).

Choose a natural number $n>1$, and let

$$
t_{i}:=\frac{i}{n}, \quad i=0, \ldots, n \text {. }
$$

We claim that there exist $x_{i} \in E, i=0, \ldots, n$, satisfying

$$
\left[x_{i}\right]=f\left(t_{i}\right), \quad i=0, \ldots, n
$$

and

$$
d\left(x_{i}, x_{i+1}\right)=\tilde{d}\left(f\left(t_{i}\right), f\left(t_{i+1}\right)\right), \quad i=0, \ldots, n-1 .
$$

Indeed, we can choose an arbitrary $x_{0} \in f(0)$ (i.e., we can pick $x_{0} \in E$ such that $\left.\left[x_{0}\right]=f(0)\right)$. Arguing inductively, suppose that we have already found $x_{i}, i=1, \ldots, j$, for some $j \geq 0$, satisfying (8) for $i=$ $1, \ldots, j$ and $(9)$ for $i=0, \ldots, j-1$ (note that this condition is empty for $j=0)$. If $j=n$ then we are done, otherwise, we let

$$
A_{j+1}:=\left\{x \in E:[x]=f\left(t_{j+1}\right) \text { and } \tilde{d}\left(\left[x_{j}\right],[x]\right)=d\left(x_{j}, x\right)\right\}
$$

(the fact that $A_{j+1}$ is nonempty easily follows from the definition of $\tilde{d})$. If we define $x_{j+1}$ to be an (arbitrarily chosen) element of $A_{j+1}$, it is clear that (8) and (9) are satisfied, respectively, for $i=j+1$ and $i=j$, and our claim is proved by induction.

Now we define

$$
g_{n}\left(t_{i}\right):=x_{i}, \quad i=0, \ldots, n .
$$

Let $l, m$ be natural numbers such that $0 \leq l<l+m \leq n$. We want to estimate the distance $d\left(x_{l}, x_{l+m}\right)$ by the modulus of continuity of $f$. For every pair $i, j$ with $0 \leq i<j \leq m$, there exists $\sigma_{i, j} \in G$ such that

$$
d\left(x_{i}, \sigma_{i, j} x_{j}\right)=\tilde{d}\left(\left[x_{l+i}\right],\left[x_{l+j}\right]\right) .
$$

By virtue of (8) and (9), we can choose $\sigma_{i, i+1}=1_{G}$, hence we can invoke Lemma 2.1. Let $i_{0}, \ldots, i_{k}$ (with $k \leq 2 \mathrm{o}(G)-1$ ) be as in (5), (6), and set

$$
\tau_{0}:=1_{G}, \quad \tau_{j}:=\sigma_{i_{0}, i_{1}} \sigma_{i_{1}, i_{2}} \cdots \sigma_{i_{j-1}, i_{j}}, \quad j=1, \ldots, k,
$$

and note that $\tau_{k}=1_{G}$ according to (6). We have then from the triangle inequality

$$
d\left(x_{l}, x_{l+m}\right)=d\left(\tau_{0} x_{l+i_{0}}, \tau_{k} x_{l+i_{k}}\right) \leq \sum_{j=0}^{k-1} d\left(\tau_{j} x_{l+i_{j}}, \tau_{j+1} x_{l+i_{j+1}}\right)=
$$




$$
=\sum_{j=0}^{k-1} d\left(\tau_{j} x_{l+i_{j}}, \tau_{j} \sigma_{i_{j}, i_{j+1}} x_{l+i_{j+1}}\right) .
$$

Using (10) we obtain

$$
\begin{gathered}
d\left(x_{l}, x_{l+m}\right) \leq \sum_{j=0}^{k-1} \tilde{d}\left(\left[x_{l+i_{j}}\right],\left[x_{l+i_{j+1}}\right]\right)=\sum_{j=0}^{k-1} \tilde{d}\left(f\left(t_{l+i_{j}}\right), f\left(t_{l+i_{j+1}}\right)\right) \leq \\
\leq \sum_{j=0}^{k-1} \omega_{f}\left(t_{l+i_{j+1}}-t_{l+i_{j}}\right) \leq \sum_{j=0}^{k-1} \omega_{f}\left(t_{l+m}-t_{l}\right) \\
\leq(2 \mathrm{o}(G)-1) \omega_{f}\left(t_{l+m}-t_{l}\right) .
\end{gathered}
$$

From the arbitrariness of $l, m$ we may write the last inequality as

$$
d\left(g_{n}\left(t_{i}\right), g_{n}\left(t_{j}\right)\right) \leq(2 \mathrm{o}(G)-1) \omega_{f}\left(\left|t_{j}-t_{i}\right|\right), \quad i, j=0, \ldots, n .
$$

Then we extend $g_{n}$ to be piecewise affine and continuous, i.e.

$$
g_{n}(t):=g_{n}\left(t_{i}\right)+n\left(t-t_{i}\right)\left(g_{n}\left(t_{i+1}\right)-g_{n}\left(t_{i}\right)\right) \quad \text { if } t \in\left(t_{i}, t_{i+1}\right)
$$

(here we exploit the linear structure of $E$ ). A standard argument then reveals that (up to a multiplicative factor in the right hand side), the estimate (11) holds everywhere on $[0,1]$, i.e.

$$
d\left(g_{n}(t), g_{n}(s)\right) \leq C_{G} \omega_{f}(|t-s|), \quad s, t \in[0,1]
$$

for some constant $C_{G}$ depending only on the order of $G$. Now let

$$
C:=\{x \in E:[x] \in f([0,1])\} .
$$

We claim that $C$ is compact. Indeed, let $\left\{x_{n}\right\} \subset C$ be a sequence. Since $f([0,1])$ is compact in $E / G$, passing to a subsequence (not relabelled) we may assume that $\left[x_{n}\right]$ converge to $[x]$ for some $x \in E$. Let $\sigma_{n} \in G$ be such that

$$
d\left(x_{n}, \sigma_{n} x\right)=\tilde{d}\left(\left[x_{n}\right],[x]\right) .
$$

Since $G$ is finite, there exists $\sigma \in G$ such that $\sigma=\sigma_{n_{k}}$, for a suitable subsequence $\left\{n_{k}\right\}$. Then we have

$$
d\left(x_{n_{k}}, \sigma x\right)=d\left(x_{n_{k}}, \sigma_{n_{k}} x\right)=\tilde{d}\left(\left[x_{n_{k}}\right],[x]\right) .
$$

Since the last term vanishes as $k \rightarrow \infty$, we obtain that $x_{n_{k}} \rightarrow \sigma x \in C$, therefore $C$ is sequentially compact hence compact.

From Theorem 2.3 below, we obtain that, up to a subsequence, $\left\{g_{n}\right\}$ converges uniformly to $g:[0,1] \rightarrow C$, and it is easy to check that $g$ satisfies $[g(t)]=f(t)$ for every $t \in[0,1]$. Moreover, the estimate (12) is maintained on passing to the limit.

Finally, the case where $(E, d)$ is a generic metric space can be reduced to the linear case by an isometric embedding argument, as follows. Let 
$E^{\prime}$ be the linear space of all real bounded functions on $E$, endowed with the sup norm, choose $x_{0} \in E$ and define $T: E \rightarrow E^{\prime}$ as

$$
(T x)(y):=d\left(x_{0}, y\right)-d(x, y), \quad \forall x, y \in E .
$$

It is easy to check (using the triangle inequality) that $T$ is an isometric embedding of $E$ into $E^{\prime}$ : then one can repeat the above construction working in $E^{\prime}$. In passing, note that it may well happen that an interpolating $g_{n}$ cannot be pulled back to $E$ : however, this is certainly true of the limit function $g$, and this concludes the proof.

Remark 2.2. We point out that (9) implies that

$$
d\left(g_{n}\left(t_{i+1}\right), g_{n}\left(t_{i}\right)\right) \leq \omega_{f}(1 / n), \quad i=0, \ldots, n-1 .
$$

In principle, this estimate concerning two adjacent points of the mesh could be used to estimate the modulus of continuity of $g_{n}$ on the whole mesh. Namely, using the triangle inequality one would immediately obtain

$$
d\left(g_{n}\left(t_{j}\right), g_{n}\left(t_{i}\right)\right) \leq|j-i| \omega_{f}(1 / n), \quad \forall i, j \in\{0, \ldots, n\} .
$$

However, this estimate does not allow one to replace the right hand side by a quantity of the kind

$$
\omega_{f}(|j-i| / n)=\omega_{f}\left(\left|t_{j}-t_{i}\right|\right)
$$

unless $\omega_{f}$ has linear growth (i.e., unless $f$ is Lipschitz continuous).

From this it appears that Lemma 2.1 is fundamental in order to obtain (11), since the constant $C_{G}$ in the right hand side does not depend on $n$.

The following Theorem is a variant of the classical Ascoli-Arzelà Theorem. The proof is omitted, since it can be carried out as usual, with only minor changes (see [7]).

Theorem 2.3 (Ascoli-Arzelà revisited). Let $I, E$ be two metric spaces with $I$ compact, and let $\left\{g_{n}\right\}$ be a sequence of continuous functions from $I$ into $E$ such that:

- $\left\{g_{n}\right\}$ is equicontinuous

- there exists a compact set $C \subseteq E$ such that, for every $\delta$-neighborhood $C_{\delta}$ of $C$, there holds $g_{n}(I) \subseteq C_{\delta}$ provided $n$ is large enough (depending on $\delta$ ).

Under the above assumptions, there exists $g: I \mapsto C$ and a subsequence $\left\{n_{k}\right\}$ such that $\left\{g_{n_{k}}\right\}$ converges to $g$, uniformly on $I$. 


\section{Continuous selections for $Q$-valued functions}

In this section we deal with multiple valued functions in $\mathbb{R}^{n}$. This is a special case of the general problem stated in the previous section, as long as we are concerned about continuity. We shall be interested, actually, in differentiability too, and this is a concept which could not be treated in the general case, due to the lack of vectorial structure in a generic metric space $E$. First of all we shall state the basic definitions for the continuity and the differentiability the way they were expressed by Almgren (see [2], [4], [5]).

Let $Q \in \mathbb{N}$.

Definition 3.1. We define by $\mathrm{Q}_{Q}\left(\mathbb{R}^{n}\right)$ the set of the unordered (and generally not distinct) $Q$-tuples of points of $\mathbb{R}^{n}$.

To be more precise, given a point $x \in \mathbb{R}^{n}$ let $[[x]]$ be the Dirac delta concentrated on $x$. Then, an element $S \in \mathbf{Q}_{Q}\left(\mathbb{R}^{n}\right)$ can be seen simply as a measure of the form

$$
S=\sum_{i=1}^{Q}\left[\left[x_{i}\right]\right], \quad x_{i} \in \mathbb{R}^{n}, i=1 \ldots, Q .
$$

Let $\Sigma_{Q}$ be the set of the permutations of $\{1, \ldots, Q\}$. Then we can define a metric on $\mathbf{Q}_{Q}\left(\mathbb{R}^{n}\right)$ by setting

$$
\mathcal{F}\left(\sum_{i=1}^{Q}\left[\left[x_{i}\right]\right], \sum_{i=1}^{Q}\left[\left[y_{i}\right]\right]\right)=\min \left\{\sum_{i=1}^{Q}\left|x_{i}-y_{\pi(i)}\right|: \pi \in \Sigma_{Q}\right\} .
$$

Remark 3.2. Let $E=\left(\mathbb{R}^{n}\right)^{Q}$. Given $x, y \in E\left(\right.$ with $x=\left(x_{1}, \ldots, x_{Q}\right)$ $\left.y=\left(y_{1}, \ldots y_{Q}\right), x_{i}, y_{i} \in \mathbb{R}^{n}\right)$, we set

$$
d(x, y)=\sum_{i=1}^{Q}\left|x_{i}-y_{i}\right|
$$

Let $G$ be the set of applications on $E$ defined this way: given a permutation $\tau \in \Sigma_{Q}$ we set $\Phi_{\tau}: E \longrightarrow E$

$$
\Phi_{\tau}\left(\left(x_{1}, \ldots, x_{Q}\right)\right)=\left(x_{\tau(1)}, \ldots x_{\tau(Q)}\right) .
$$

It is clear that $d\left(\Phi_{\tau}(x), \Phi_{\tau}(y)\right)=d(x, y)$, hence $G=\left\{\Phi_{\tau}: \tau \in \Sigma_{Q}\right\}$ is a group of isometries of $E$. We can identify the quotient space $E / G$ and $Q_{Q}\left(\mathbb{R}^{n}\right)$ with the natural isomorphism $[x] \longleftrightarrow \sum_{i=1}^{Q}\left[\left[x_{i}\right]\right]$ where $[x]$ is the orbit of $x$ (see $(2))$. The metric $\tilde{d}$ coincides with $\mathcal{F}$. This somewhat redundant notation is motivated by the effort to keep the same notations of some previous works on multiple valued functions in the Euclidean case (see for example [2] and [12]). 
If $A \subset \mathbb{R}^{m}$ and $f: A \longrightarrow \mathrm{Q}_{Q}\left(\mathbb{R}^{n}\right)$ we say that $f$ is a $Q$-valued function on $A$; the continuity of $f$ has to be intended with respect to the metric $\mathcal{F}$ on $\mathbf{Q}_{Q}\left(\mathbb{R}^{n}\right)$. Given a $Q$-valued function $f: A \longrightarrow \mathbf{Q}_{Q}\left(\mathbb{R}^{n}\right)$ we can find $Q$ functions $f_{i}: A \longrightarrow \mathbb{R}^{n}$ such that $f(x)=\sum_{i=1}^{Q}\left[\left[f_{i}(x)\right]\right]$.

Definition 3.3. Let $A \subset \mathbb{R}^{m}$ and $f: A \longrightarrow \mathrm{Q}_{Q}\left(\mathbb{R}^{n}\right)$ be a $Q$-valued function. We say that $f$ is Lipschitz if there exists $c>0$ such that

$$
\mathcal{F}(f(x), f(y)) \leq c|x-y| \quad \forall x, y \in A .
$$

We say that $f$ is Hölder continuous if there exist $\lambda>0,0<\alpha<1$ such that

$$
\mathcal{F}(f(x), f(y)) \leq \lambda|x-y|^{\alpha} \quad \forall x, y \in A .
$$

Definition 3.4. Let $A \subset \mathbb{R}^{m}$ be an open set and $f: A \longrightarrow \mathrm{Q}_{Q}\left(\mathbb{R}^{n}\right)$ a $Q$-valued function. Given $x \in A$ we say that $f$ can be affinely approximated at $x$ if there exist linear functions $L_{i}(x): \mathbb{R}^{m} \longrightarrow \mathbb{R}^{n}, i=$ $1, \ldots, Q$ such that, if we set

$$
A f(x)(y)=\sum_{i=1}^{Q}\left[\left[f_{i}(x)+L_{i}(x)(y-x)\right]\right]
$$

it results

$$
\lim _{y \rightarrow x} \frac{\mathcal{F}(f(y), A f(x)(y))}{|y-x|}=0 .
$$

If $f$ can be affinely approximated at each point $x \in A$ we say that $f$ can be affinely approximated in $A$. We call the $Q$-valued function $L=\sum_{i=1}^{Q}\left[\left[L_{i}\right]\right]$ the derivative of $f$.

Remark 3.5. Let $A \subset \mathbb{R}^{m}$ be an open set and $f: A \longrightarrow \mathbf{Q}_{Q}\left(\mathbb{R}^{m}\right)$ be affinely approximatable in $A$ with derivative $L$. Then $f$ is continuous in $A$.

Definition 3.6. We say that a $Q$-valued function $f: A \longrightarrow \mathbf{Q}_{Q}\left(\mathbb{R}^{n}\right)$, $A \subset \mathbb{R}^{m}$, is $C^{1}(A)$ if $f$ is affinely approximatable with derivative $L$ and the multiple valued function $G: A \rightarrow \mathbf{Q}_{Q}\left(\mathbb{R}^{n} \times \mathbb{R}^{m n}\right)$ given by

$$
x \rightarrow G(x)=\sum_{i=1}^{Q}\left[\left[\left(f_{i}, L_{i}\right)\right]\right]
$$

is continuous. Moreover we say that $f$ is $C^{1, \alpha}(A)$ if there exists a constant $c>0$ such that, for each $x, y \in A$ it results

$$
\min \left\{\sum_{i=1}^{Q} \frac{\left|f_{i}(x)-f_{\pi(i)}(y)\right|}{|x-y|}+\frac{\left\|L_{i}(x)-L_{\pi(i)}(y)\right\|}{|x-y|^{\alpha}}: \pi \in \Sigma_{Q}\right\} \leq c,
$$

where $\left\|L_{i}(x)\right\|$ is the norm of the linear functional $L_{i}(x): \mathbb{R}^{m} \longrightarrow \mathbb{R}^{n}$. 
In the same way we can define the class $C^{k, \alpha}(A)$. The question is if for a $Q$-valued function $f$ it is possible to choose the functions $f_{i}$ in such a way that each $f_{i}$ is regular in an ordinary sense. The answer is, in general, negative (see [12]). We will show that this is possible in some special cases.

Definition 3.7. Let $A \subset \mathbb{R}^{m}$ and $f: A \longrightarrow \mathbf{Q}_{Q}\left(\mathbb{R}^{n}\right)$ be a continuous $Q$-valued function. If there exist continuous functions $g_{i}: A \longrightarrow \mathbb{R}^{n}$, $i=1, \ldots, Q$ such that $f(x)=\sum_{i=1}^{Q}\left[\left[g_{i}(x)\right]\right], \forall x \in A$ then we say that the vector $\left(g_{1}, \ldots, g_{Q}\right)$ is a continuous selection for $f$. To simplify notation we will refer to $g=\sum_{i=1}^{Q}\left[\left[g_{i}\right]\right]$ as the $Q$-valued function that is a continuous selection of $f$. (Of course $g$ and $f$ are the same multivalued function: by abuse of notation we are distinguishing two different selections.)

Definition 3.8. If $f$ is Hölder (Lipschitz) continuous and $g_{i}$ is Hölder (Lipschitz) continuous for every $i$, we say that $g$ is an Hölder (Lipschitz) selection for $f$. Moreover, if $f$ is also affinely approximatable with derivative $L$, we define $M_{i}(x)=L_{\pi_{x}(i)}(x)$, where $\pi_{x} \in \Sigma_{Q}$ is any permutation such that $g_{i}(x)=f_{\pi_{x}(i)}(x)$. Of course if we set

$$
A g(x)(y)=\sum_{i=1}^{Q}\left[\left[g_{i}(x)+M_{i}(x)(y-x)\right]\right]
$$

then $\operatorname{Ag}(x)(y)=A f(x)(y)$. Again by abuse of notation, even if $M(x)=$ $\sum_{i=1}^{Q}\left[\left[M_{i}(x)\right]\right]$ is the same multivalued function of $L$, we call $M$ the derivative of $g$.

Remark 3.9. The choice of the permutation $\pi_{x}$ is clearly not unique but, for our purpose, we do not have to care about this. Nevertheless, it is possible to fix any rule for the choice of $\pi_{x}$, for example:

$$
\left\{\begin{array}{c}
\pi_{x}(1)=\min \left\{j: f_{j}(x)=g_{1}(x)\right\} \\
\pi_{x}(k+1)=\min \left\{j: f_{j}(x)=g_{k+1}(x), j \neq \pi_{x}(i) \forall i=1, \ldots, k\right\} \\
\quad \text { for } k=1, \ldots Q-1 .
\end{array}\right.
$$

As we will see in section 4, using the result of the previous section, when $f$ is a $Q$-valued differentiable function with derivative $L$ we are able to make a continuous selection $g$ of $f$ and a continuous selection $M$ of $L$. But a priori this does not mean that the $M_{i}$ are the derivatives of the $g_{i}$. However the statement is true and we will prove it after several lemmas.

Theorem 3.10. Let $A \subset \mathbb{R}^{m}$ and $f: A \longrightarrow \mathrm{Q}_{Q}\left(\mathbb{R}^{n}\right)$ be a $Q$-valued function affinely approximatable with continuous derivative $L$. If there exists a $Q$-valued function $g$, affinely approximatable with derivative 
$M$, such that $g$ is a continuous selection for $f$ and $M$ is a continuous selection for $L$, then the functions $g_{i}: A \longrightarrow \mathbb{R}^{n}$ are of class $C^{1}$ and $d g_{i}(x)=M_{i}(x)$ for every $x \in A$ and for every $i=1, \ldots, Q$.

Lemma 3.11. Let $A \subset \mathbb{R}^{m}$ and $f: A \longrightarrow \mathbf{Q}_{Q}\left(\mathbb{R}^{n}\right)$ be a continuous $Q$-valued function and let $g$ be a continuous selection for $f$. Fix $x \in$ $A, i \in\{1, \ldots, Q\}$ and set $I_{i}(x)=\left\{j \in\{1, \ldots, Q\}: g_{j}(x)=g_{i}(x)\right\}$. For $y \neq x$ let $\pi_{x}^{y} \in \Sigma_{Q}$ be any permutation which attains the following minimum:

$$
\min _{\pi \in \Sigma_{Q}} \sum_{j=1}^{Q}\left|g_{j}(x)+M_{j}(x)(y-x)-g_{\pi(j)}(y)\right|:=\mathcal{F}(A g(x)(y), g(y)) .
$$

Then, if $g$ is affinely approximatable at $x$, there exists $\delta>0$ such that

$$
\pi_{x}^{y}\left(I_{i}(x)\right)=\left(\pi_{x}^{y}\right)^{-1}\left(I_{i}(x)\right)=I_{i}(x) \quad \forall y \in A:|x-y|<\delta .
$$

Moreover, there exists $\delta^{\prime}>0$ such that for every $z \in B_{\delta^{\prime}}(x) \cap A$ it results

$$
I_{i}(x)=\bigcup_{j \in I_{i}(x)} I_{j}(z)
$$

Proof. Let us choose an arbitrary $k \in I_{i}(x)$. By contradiction, let $\left(y_{n}\right) \subset A$ be a sequence converging to $x$ such that $\pi_{x}^{y_{n}}(k) \notin I_{i}(x), \forall n \in$ $\mathbb{N}$. Using the continuity of every $g_{j}$ we have that there exists $\varepsilon>0$ such that, for $n$ large enough,

$$
\left|g_{k}(x)-g_{\pi_{x}^{y_{n}(k)}}\left(y_{n}\right)\right|=\left|g_{i}(x)-g_{\pi_{x}^{y_{n}}(k)}\left(y_{n}\right)\right|>\varepsilon \text {. }
$$

Hence,

$$
\begin{gathered}
\mathcal{F}\left(A g(x) y_{n}, g\left(y_{n}\right)\right) \geq \frac{\left|g_{k}(x)+M_{k}(x)\left(y_{n}-x\right)-g_{\pi_{x}^{y_{n}(k)}}\left(y_{n}\right)\right|}{\left|y_{n}-x\right|} \\
\geq \frac{\varepsilon}{\left|y_{n}-x\right|}-\left\|M_{k}(x)\right\| \longrightarrow \infty \quad \text { when } n \rightarrow \infty
\end{gathered}
$$

which gives the contradiction because $g$ is affinely approximatable at $x$. Then, there exists $\delta_{k}>0$ such that $\pi_{x}^{y}(k) \in I_{i}(x)$ if $|y-x|<\delta_{k}$. If we set $\delta=\min \left\{\delta_{j}: j \in I_{i}(x)\right\}$ then $\pi_{x}^{y}\left(I_{i}(x)\right) \subset I_{i}(x)$ for every $y \in A$ such that $|y-x|<\delta$. The opposite inclusion and the equality $\left(\pi_{x}^{y}\right)^{-1}\left(I_{i}(x)\right)=I_{i}(x)$ follow from the invertibility of $\pi_{x}^{y}$.

By the continuity of every $g_{k}$ we know that there exists $\delta^{\prime}>0$ such that if $|z-x|<\delta^{\prime}$ then $g_{k}(z) \neq g_{j}(z)$ for every $j \in I_{i}(x)$ and for every $k \notin I_{i}(x)$. Let us choose arbitrary $j \in I_{i}(x), z \in B_{\delta^{\prime}}(x) \cap A$ and $k \in I_{j}(z)$. If it was $k \notin I_{i}(x)$ then $g_{k}(z) \neq g_{j}(z)$, which contradicts the fact that $k \in I_{j}(z)$. Hence $I_{j}(z) \subset I_{i}(x)$. On the other side $j \in I_{j}(z)$ for every $j \in I_{i}(x)$, hence $I_{i}(x) \subset \bigcup_{j \in I_{i}(x)} I_{j}(z)$. 
Corollary 3.12. Let $A \subset \mathbb{R}^{m}$ and $f: A \longrightarrow \mathrm{Q}_{Q}\left(\mathbb{R}^{n}\right)$ be continuous and affinely approximatable and let $g$ be any continuous selection affinely approximatable for $f$, with derivative $M$. If $x \in A$ and $i \in\{1, \ldots, Q\}$ are such that $g_{i}(x) \neq g_{j}(x)$ for every $j \neq i$, then $g_{i}$ is differentiable at $x$ and $d g_{i}(x)=M_{i}(x)$.

Corollary 3.13. Let $A \subset \mathbb{R}^{m}, f: A \longrightarrow \mathrm{Q}_{Q}\left(\mathbb{R}^{n}\right)$ a continuous and affinely approximatable $Q$-valued function and $g: A \longrightarrow \mathrm{Q}_{Q}\left(\mathbb{R}^{n}\right)$ be a continuous affinely approximatable selection for $f$ as in Definition 3.8. Let us fix $x \in A$ and $i \in\{1, \ldots, Q\}$. For $k \in\{1, \ldots, m\}$ we shall use the notation $M_{j}^{k}$ for the $k$-th column of the matrix $M_{j}$. Then if $M_{j}^{k}(x)=M_{i}^{k}(x)$ for every $j \in\{1, \ldots, Q\}$ such that $g_{j}(x)=g_{i}(x)$, then there exists $\frac{\partial g_{i}}{\partial x_{k}}(x)=M_{i}^{k}(x)$.

Proof. Let $I_{i}(x)$ and $\pi_{x}^{y}$ be as in Lemma 3.11. It follows that there exists $\delta>0$ such that if $|y-x|<\delta$ then $g_{\pi_{x}^{y}(i)}(x)=g_{i}(x)$, hence $M_{\left(\pi_{x}^{y}\right)^{-1}(i)}^{k}(x)=M_{i}^{k}(x)$. If we call $\left\{e_{1}, \ldots, e_{m}\right\}$ the canonical base of $\mathbb{R}^{m}$, it follows easily that:

$$
\lim _{h \rightarrow 0} \frac{\left|g_{i}(x)+M_{i}^{k}(x) h-g_{i}\left(x+h e_{k}\right)\right|}{|h|}=0 .
$$

\section{Proof of Theorem 3.10}

We will prove the theorem by induction on $Q$.

We remind that the functions $g_{k}$ and $M_{k}$ are continuous, hence, we only need to prove that every $g_{k}$ is differentiable and $d g_{k}=M_{k}$. If $Q=1$ there is nothing to prove. Now we suppose the thesis true for

$Q \leq K$ and we will prove it for $Q=K+1$. Let $f: A \longrightarrow \mathrm{Q}_{K+1}\left(\mathbb{R}^{n}\right)$ be a $C^{1}$ function and let $g$ and $M$ as in the hypothesis of the theorem. Let us fix $x \in A$. We will distinguish two cases:

a) there exist $i, j \in\{1, \ldots, K+1\}$ such that $g_{i}(x) \neq g_{j}(x)$;

b) $g_{1}(x)=\cdots=g_{K+1}(x)$.

In case a) let $\pi_{x}^{y}$ be as in Lemma 3.11. It follows that there exists $\delta>0$ such that, if $y \in A$ and $|y-x|<\delta$ then $\pi_{x}^{y}\left(I_{i}(x)\right)=\left(\pi_{x}^{y}\right)^{-1}\left(I_{i}(x)\right)=$ $I_{i}(x)$. If we set $J_{i}(x)=\{1, \ldots, K+1\} \backslash I_{i}(x)$, then we have $\pi_{x}^{y}\left(J_{i}(x)\right)=$ $\left(\pi_{x}^{y}\right)^{-1}\left(J_{i}(x)\right)=J_{i}(x)$. We can split the functions $g_{k}$ into two groups in order to create the following multiple valued functions:

$$
h(y)=\sum_{k \in I_{i}(x)}\left[\left[g_{k}(y)\right]\right], \quad \varphi(y)=\sum_{k \in J_{i}(x)}\left[\left[g_{k}(y)\right]\right]
$$

defined in the neighborhood of $x, B_{\delta}(x) \cap A$. We will prove that $h$ and $\varphi$ are $C^{1}$ multiple valued functions. 
The first thing we prove is that $h$ is affinely approximatable in a neighborhood of $x$. From Lemma 3.11 there exists $\delta^{\prime} \leq \delta$ such that $I_{i}(x)=\bigcup_{j \in I_{i}(x)} I_{j}(z)$ for every $z \in B_{\delta^{\prime}}(x) \cap A$. Then, for every $y$ in a neighborhood of $z$, it results:

$$
\pi_{z}^{y}\left(I_{i}(x)\right)=\bigcup_{j \in I_{i}(x)} \pi_{z}^{y}\left(I_{j}(z)\right)=\bigcup_{j \in I_{i}(x)} I_{j}(z)=I_{i}(x) .
$$

Hence, if we define $\sigma_{I_{i}(x)}$ as the set of all the permutations over $I_{i}(x)$, we have:

$$
\begin{gathered}
\lim _{y \rightarrow z} \frac{\mathcal{F}(A h(z)(y), h(y))}{|y-z|}= \\
=\lim _{y \rightarrow z} \frac{\min \left\{\sum_{k \in I_{i}(x)}\left|g_{k}(z)+M_{k}(z)(y-z)-g_{\pi(k)}(y)\right|: \pi \in \sigma_{I_{i}(x)}\right\}}{|y-z|} \\
\leq \lim _{y \rightarrow z} \frac{\sum_{k \in I_{i}(x)}\left|g_{k}(z)+M_{k}(z)(y-z)-g_{\pi_{z}(k)}(y)\right|}{|y-z|}=0
\end{gathered}
$$

because $g$ is affinely approximatable at $z$. Hence $h$ is affinely approximatable at $z$ for every $z \in B_{\delta^{\prime}} \cap A$. Of course, from the continuity of $g_{i}$ and $M_{i}$ it follows that the map

$$
h(y)=\sum_{k \in I_{i}(x)}\left[\left[\left(g_{k}(y), M_{k}(y)\right)\right]\right]
$$

is continuous. Hence we have proved that $h$ is a $C^{1} Q$-valued function with $Q \leq K$ and the induction hypothesis tells us that the $g_{k}$, with $k \in I_{i}(x)$ are of class $C^{1}$ in $B_{\delta^{\prime}}(x)$. The same result holds true for $\varphi$. Hence we have obtained that $g_{k}$ is differentiable at $x$ for every $k=1, \ldots, K+1$ and $d g_{k}(x)=M_{k}(x)$.

Now examine case b). Let us fix $k \in\{1, \ldots, m\}$. There are two possibilities:

b1) there exists $\delta>0$ such that for every $h \in \mathbb{R}$ with $0<|h|<\delta$ there exist $i, j$ such that $g_{i}\left(x+h e_{k}\right) \neq g_{j}\left(x+h e_{k}\right)$;

b2) there exists a sequence of real numbers $h_{n} \longrightarrow 0$ such that

$$
g_{1}\left(x+h_{n} e_{k}\right)=\cdots=g_{K+1}\left(x+h_{n} e_{k}\right) .
$$

In case b1) we can apply the result in a) to the points $x+h e_{k}$, obtaining that every $g_{i}$ has partial derivative with respect to $x_{k}$ on the open segments joining $x$ to $x+\delta e_{k}$ and $x$ to $x-\delta e_{k}$. Then there exists $\eta$ depending on $h$, with $|\eta|<|h|$, such that:

$$
\frac{\partial g_{i}}{\partial x_{k}}(x)=\lim _{h \rightarrow 0} \frac{g_{i}\left(x+h e_{k}\right)-g_{i}(x)}{h}=\lim _{h \rightarrow 0} \frac{\partial g_{i}}{\partial x_{k}}\left(x+\eta e_{k}\right)=
$$




$$
=\lim _{h \rightarrow 0} M_{i}^{k}\left(x+\eta e_{k}\right)=M_{i}^{k}(x) \quad i=1, \ldots K+1 .
$$

In case b2) we set $y_{n}=x+h_{n} e_{k}$. Then:

$$
\begin{gathered}
0=\lim _{n \rightarrow \infty} \frac{\mathcal{F}\left(A g(x)\left(y_{n}\right), g\left(y_{n}\right)\right)}{\left|h_{n}\right|}= \\
=\lim _{n \rightarrow \infty} \frac{\sum_{i=1}^{K+1}\left|g_{i}(x)+M_{i}(x) h_{n} e_{k}-g_{\pi_{x}^{y_{n}(i)}}\left(y_{n}\right)\right|}{\left|h_{n}\right|}= \\
=\lim _{n \rightarrow \infty} \sum_{i=1}^{K+1} \frac{\left|g_{1}(x)+M_{i}^{k}(x) h_{n}-g_{1}\left(y_{n}\right)\right|}{\left|h_{n}\right|} .
\end{gathered}
$$

Hence $M_{1}^{k}(x)=\cdots=M_{K+1}^{k}(x)$ and Corollary 3.13 gives us the existence of $\frac{\partial g_{i}}{\partial x_{k}}(x)$ for $i=1, \ldots, K+1$.

In both cases there exist the derivatives $\frac{\partial g_{i}}{\partial x_{k}}(x)=M_{i}^{k}(x)$ for every $k=1, \ldots, m$. Then the continuity of $M_{i}^{k}$ gives us the differentiability of each $g_{i}$ in $x$. This concludes the proof.

\section{Vectorial $Q$-VAlued CuRVes}

In this section we examine what happens when $m=1$. We shall prove that, in this case and under suitable regularity hypothesis, the graph of a multiple valued function is actually the finite union of curves in $\mathbb{R}^{n+1}$.

Theorem 4.1. Let $[a, b] \subset \mathbb{R}$ and $f:[a, b] \longrightarrow \mathbf{Q}_{Q}\left(\mathbb{R}^{n}\right), f(x)=$ $\sum_{i=1}^{Q}\left[\left[f_{i}(x)\right]\right]$ be a $C^{\alpha} Q$-valued function. Then there exists a $C^{\alpha}$ selection for $f$.

Proof. For simplicity let $[a, b]=[0,1]$. We are going to apply Theorem 1.1. We define $E, G$ and $\tilde{d}$ as in Remark 3.2 and we set $\tilde{f}:[0,1] \longrightarrow$ $E / G$ as

$$
\tilde{f}(t)=\left[\left(f_{i}(t), \ldots, f_{Q}(t)\right)\right]
$$

Then we have that:

$$
\tilde{d}(\tilde{f}(t), \tilde{f}(s))=\mathcal{F}\left(\sum_{i=1}^{Q}\left[\left[f_{i}(t)\right]\right], \sum_{i=1}^{Q}\left[\left[f_{i}(s)\right]\right]\right) \leq c|t-s|^{\alpha} .
$$

Hence $\tilde{f}$ is Hölder continuous and $\omega_{\tilde{f}}(\delta) \leq c \delta^{\alpha}$. Then, by Theorem 1.1 there exists a lifting $g:[0,1] \longrightarrow E$ such that $g$ is Hölder continuous with exponent $\alpha$. This completes the proof. 
Theorem 4.2. Let $f:[a, b] \longrightarrow \mathbf{Q}_{Q}\left(\mathbb{R}^{n}\right)$ be a $C^{1, \alpha} Q$-valued function. Then there exist functions $g_{i}:[a, b] \longrightarrow \mathbb{R}^{n}$ such that $g_{i} \in C^{1, \alpha}([a, b])$ for $i=1, \ldots, Q$ and $f(x)=\sum_{i=1}^{Q}\left[\left[g_{i}(x)\right]\right]$.

Proof. Once again we suppose $[a, b]=[0,1]$.

Let us join together $f$ and its derivative to obtain a vectorial function with $2 n$ components:

$$
F:[a, b] \longrightarrow \mathbf{Q}_{Q}\left(\mathbb{R}^{2 n}\right), \quad F(x)=\sum_{i=1}^{Q}\left[\left[f_{i}(x), L_{i}(x)\right]\right] .
$$

We observe that $F$ is a $C^{\alpha}$ multiple valued function, indeed

$$
\begin{gathered}
\mathcal{F}(F(x), F(y))=\min \left\{\sum_{i=1}^{Q}\left|F_{i}(x)-F_{\pi(i)}(y)\right|: \pi \in \Sigma_{Q}\right\} \leq \\
\leq|x-y|^{\alpha} \min \left\{\sum_{i=1}^{Q} \frac{\left|f_{i}(x)-f_{\pi(i)}(y)\right|}{|x-y|}+\frac{\left|L_{i}(x)-L_{\pi(i)}(y)\right|}{|x-y|^{\alpha}}: \pi \in \Sigma_{Q}\right\} .
\end{gathered}
$$

Then we can apply Theorem 4.1 to obtain a Hölder selection $G$ for $F$. Let $G(x)=\sum_{i=1}^{Q}\left[\left[\left(g_{i}(x), M_{i}(x)\right)\right]\right]$. Observing that $\mathcal{F}(g(x), g(y)) \leq$ $\mathcal{F}(G(x), G(y))$ we have that $g$ is a Hölder selection for $f$. The same result holds for $M$ which is a Hölder selection for $L$. It is easy to prove that $g$ is affinely approximatable with derivative $M$. Hence we can apply Theorem 3.10 to obtain that $g_{i} \in C^{1}([a, b])$ for every $i=1, \ldots, Q$. Since $g_{i}^{\prime}(x)=M_{i}(x)$ is Hölder continuous, then $g_{i} \in C^{1, \alpha}([a, b])$ for every $i=1, \ldots, Q$.

In the same way we can prove that a $C^{k, \alpha} Q$-valued function of real variable admits a $C^{k, \alpha}$ selection.

\section{Scalar $Q$-Valued Functions}

In this last section we examine the case $n=1$. In this situation we obtain a different result with respect to vectorial curves. In fact we are not able to prove the differentiability.

Theorem 5.1. Let $A \subset \mathbb{R}^{m}$. If $f: A \longrightarrow \mathbf{Q}_{Q}(\mathbb{R})$ is continuous then there exist continuous functions $g_{i}: A \longrightarrow \mathbb{R}, i=1, \ldots, Q$, such that $f(x)=\sum_{i=1}^{Q}\left[\left[g_{i}(x)\right]\right], \forall x \in A$.

Proof. We shall prove the thesis by induction. If $Q=1$ there is nothing to prove. Let $Q \geq 2$. We can write $f(x)=\sum_{i=1}^{Q}\left[\left[f_{i}(x)\right]\right], \forall x \in A$. We are going to show that it is possible to sort the functions $f_{i}$ to obtain the continuity. Let us define

$$
g(x)=\max \left\{f_{i}(x): i=1, \ldots, Q\right\}
$$




$$
I(x)=\max \left\{i \in\{1, \ldots Q\}: f_{i}(x)=g(x)\right\} .
$$

We want to show that $g$ is continuous. Let us fix $x, y \in A$; then $g(x)=f_{I(x)}(x), g(y)=f_{I(y)}(y)$. If $g(x) \geq g(y)$ then

$|g(x)-g(y)|=f_{I(x)}(x)-f_{I(y)}(y) \leq f_{I(x)}(x)-f_{k}(y) \quad \forall k \in\{1, \ldots, Q\}$.

Hence, for every $\pi \in \Sigma_{Q}$ we have:

$$
|g(x)-g(y)| \leq f_{I(x)}(x)-f_{\pi(I(x))}(y) \leq \sum_{i=1}^{Q}\left|f_{i}(x)-f_{\pi(i)}(y)\right|
$$

and, taking the minimum on $\Sigma_{Q}$ :

$$
|g(x)-g(y)| \leq \mathcal{F}(f(x), f(y)) .
$$

The same results holds true if $g(x) \leq g(y)$, therefore $g$ is continuous. The next step is to show that the $(Q-1)$-valued function obtained "subtracting" $g$ from $f$ is continuous (with the metric on $\mathbf{Q}_{Q-1}(\mathbb{R})$ ). Let $\tilde{f}(x)=\sum_{i \neq I(x)}\left[\left[f_{i}(x)\right]\right]$. Given $x, y \in A$ we fix a permutation $\pi \in \Sigma_{Q}$.

If $\pi(I(x))=I(y)$ then we define a one-to-one function

$$
\alpha:\{i: 1 \leq i \leq Q, i \neq I(x)\} \longrightarrow\{j: 1 \leq j \leq Q, j \neq I(y)\}
$$

by $\alpha(i)=\pi(i)$. Then

$$
\sum_{i=1}^{Q}\left|f_{i}(x)-f_{\pi(i)}(y)\right| \geq \sum_{i \neq I(x)}\left|f_{i}(x)-f_{\alpha(i)}(y)\right| \geq \mathcal{F}(\tilde{f}(x), \tilde{f}(y)) .
$$

If $\pi(I(x)) \neq \pi(I(y))$ then we claim that

$$
\begin{gathered}
\left|f_{I(x)}(x)-f_{\pi(I(x))}(y)\right|+\left|f_{\pi^{-1}(I(y))}(x)-f_{I(y)}(y)\right| \geq \\
\geq\left|f_{\pi^{-1}(I(y))}(x)-f_{\pi(I(x))}(y)\right| .
\end{gathered}
$$

Indeed, if $f_{\pi^{-1}(I(y))}(x) \leq f_{\pi(I(x))}(y)$ then

$$
\begin{aligned}
& \left|f_{\pi^{-1}(I(y))}(x)-f_{\pi(I(x))}(y)\right|=f_{\pi_{(I(x))}}(y)-f_{\pi^{-1}(I(y))}(x) \leq \\
& \leq f_{I(y)}(y)-f_{\pi^{-1}(I(y))}(x) \leq\left|f_{\pi^{-1}(I(y))}(x)-f_{I(y)}(y)\right| .
\end{aligned}
$$

With the same argument, if $f_{\pi(I(x))}(y) \leq f_{\pi^{-1}(I(y))}(x)$ then

$$
\left|f_{\pi^{-1}(I(y))}(x)-f_{\pi(I(x))}(y)\right| \leq\left|f_{I(x)}(x)-f_{\pi(I(x))}(y)\right| .
$$

From (14) and (15) follow (13) hence, we can define

$$
\begin{cases}\alpha(k)=\pi(k) & \text { for } k \neq I(x), \pi^{-1}(I(y)) \\ \alpha\left(\pi^{-1}(I(y))=\pi(I(x))\right. & \end{cases}
$$


$\alpha$ is well defined, one-to-one and

$$
\sum_{i=1}^{Q}\left|f_{i}(x)-f_{\pi(i)}(y)\right| \geq \sum_{i \neq I(x)}\left|f_{i}(x)-f_{\alpha(i)}(y)\right| \geq \mathcal{F}(\tilde{f}(x), \tilde{f}(y)) .
$$

Taking the minimum on $\pi \in \Sigma_{Q}$ we have:

$$
\mathcal{F}(f(x), f(y)) \geq \mathcal{F}(\tilde{f}(x), \tilde{f}(y))
$$

hence $\tilde{f}$ is a continuous $(Q-1)$-valued function. This completes the proof.

Acknowledgments. We wish to thank Professor Luigi Ambrosio for his kind help.

\section{REFERENCES}

[1] F. J. Almgren, Jr.: Dirichlet's problem for multiple valued functions and the regularity of mass minimizing integral currents, in Minimal Submanifolds and Geodesics (Proc. Japan-United States sem., Tokyo, 1977), North-Holland, Amsterdam - New York, (1979), 1-6.

[2] F. J. Almgren, Jr.: Approximation of rectifiable currents by Lipschitz Q valued functions. Seminar on minimal submanifolds, E. Bombieri ed., Annals of mathematics studies, 103, Princeton University Press (1983), 243-259.

[3] F. J. Almgren: $Q$ valued functions minimizing Dirichlet's integral and the regularity of area minimizing rectifiable currents up to codimension two, Bull. Amer. Math. Soc. (N.S.), 8 (1983), no. 2, 327-328.

[4] F. J. Almgren: Deformation and multiple-valued functions, in Geometric measure theory and the calculus of variations (Arcata, Calif., 1984), W. K. Allard and F. J. Almgren eds., Proc. Sympos. Pure Math. 44 Amer. Math. Soc., Providence, R.I. (1986), 29-130.

[5] F. J. Almgren \& B. Super: Multiple valued functions in the geometric calculus of variations, in Variational methods for equilibrium problems of fluids (Trento, 1983), Astérisque No. 118, Société Mathématique de France (1984), 13-32.

[6] L. Ambrosio, M. Gobbino \& D. Pallara: Approximation problems for curvature varifolds, J. Geom. Anal. 8, no. 1 (1998), 1-19.

[7] L. Ambrosio \& P. Tilli: Selected topics on "Analysis in Metric Spaces", Scuola Normale Superiore, Pisa (2000), in press.

[8] E. De Giorgi: Introduzione ai problemi con discontinuità libere, in Symmetry in nature: a volume in honour of L. A. Radicati di Brozolo, I, Scuola Normale Superiore, Pisa (1989), 265-285.

[9] E. De Giorgi: Free discontinuity problems in calculus of variations, in Frontiers of pure and applied mathematics, a collection of papers dedicated to J. L. Lions on the occasion of his $60^{\text {th }}$ birthday, R. Dautray ed., North-Holland, Amsterdam (1991), 55-62.

[10] H. Federer: Geometric measure theory, Springer, Berlin (1969).

[11] C. R. Grisanti: On a functional depending on curvature and edges, to appear in Rend. Sem. Mat. Univ. Padova 105 (2001). 
[12] J. E. Hutchinson: $C^{1, \alpha}$ multiple function regularity and tangent cone behaviour for varifolds with second fundamental form in $L^{p}$, in Geometric measure theory and the calculus of variations (Arcata, Calif., 1984), W.K.Allard and F.J.Almgren eds., Proc. Symp. Pure Math. 44 Ann. Math. Soc., Providence (1986), 281-306.

[13] C. Mantegazza: Curvature Varifolds with boundary, J. Differential Geom. 43 (1996), no. 4, 807-843.

[14] P. Mattila: Lower semicontinuity, existence and regularity theorems for elliptic variational integrals of multiple valued functions, Trans. Amer. Math. Soc. 280 (1983), no. 2, 589-610.

[15] L. Simon: Lectures on geometric measure theory, Proceedings of the Centre for Mathematical Analysis, Australian National University, 3. Australian National University, Centre for Mathematical Analysis, Canberra, 1983.

[16] B. Solomon: A new proof of the closure theorem for integral currents, Indiana Univ. Math. J. 33 (1984), no. 3, 393-418. 